\title{
Adverse Childhood Experiences in a Group of Inpatient Treatment-Seeking Individuals with Prescription Opioid Use Disorder
}

\author{
Suchismita Ray* \\ Department of Health Informatics, School of Health Professions, Rutgers, The State University of New Jersey, Newark, NJ, USA
}

*Corresponding author: Suchismita Ray, Ph.D, Department of Health Informatics, School of Health Professions, Rutgers, The State University of New Jersey, 65 Bergen Street, Newark, NJ, USA.

To Cite This Article: Suchismita Ray. Adverse Childhood Experiences in a Group of Inpatient Treatment-Seeking Individuals with Prescription Opioid Use Disorder. Am J Biomed Sci \& Res. 2019 - 5(6). AJBSR.MS.ID.000975. DOI: 10.34297/AJBSR.2019.05.000975.

Received: 門 October 3, 2019; Published: 眥 October 24, 2019

\section{Commentary}

Prescription opioid (PO) addiction is a critical health problem both in the U.S. and internationally [1]. There were 18,893 overdose deaths related to PO pain relievers in 2014 in the U.S. alone [2].The costs of U.S. PO epidemic are estimated at $\$ 78.5$ billion [3] and are rapidly increasing with increasing PO use. In 2015, 276,000 adolescents were current nonmedical users of pain relievers with 122,000 having an addiction to prescription pain relievers [4]. From 2002 to 2011, there was a 1.9-fold increase in the total number of deaths involving POs [5]. The current PO epidemic necessitates a fuller understanding of the risk factors for PO initiation. Doing so will provide us the opportunity to enhance prevention efforts and to reduce mortality by intervening at a much younger age. A small body of research has indicated an association between adverse childhood experiences (ACEs) and non-medical PO use [6]. Neuroscience suggests that ACEs may guide to physiological processes that cause disruption in early development of the central nervous system, diminishing ability to cope with emotions [7], and leading to impaired emotional and behavioral self-regulation over the life course [8]. And as a result, individuals may self-medicate with substances to cope with the dysregulated stress response $[9,10]$.

This article reports the ACEs in a group of 26 (six females) longterm inpatient treatment-seeking individuals with prescription opioid use disorder and 19 (10 females) healthy controls between the ages of 21 and 54 years. The ACEs were collected using the Childhood Experience (ACE) questionnaire [11] to obtain any history of physical, emotional, or sexual trauma, parental incarceration, threatened with violence, or parental substance abuse during the first 18 years of life. In addition, any current stress related affective disorders (e.g., anxiety, depression or PTSD) and perceived stress scores (Perceived Stress Scale; [12]) are also assessed in these two groups. ACEs were reported significantly higher in PO patients compared to the control participants (t-test, $\mathrm{p}$ $<.001$ ). Approximately $80 \%$ of individuals with prescription opioid use disorder experienced adverse childhood events. Therefore, there is a strong association between ACEs and PO addiction in the later life. Of the PO patients who had ACEs, $65 \%$ of them reported three or more adverse childhood events. Controls reported very low ACEs score, approximately 0.58 , and only one participant reported ACEs score above 3. In the PO sample, the PO initiation occurred during the emerging or early adulthood and thus after the exposure to the adverse childhood events.

In addition, 13 of the 26 individuals with prescription opioid use disorder $(50 \%)$ were suffering from stress related affective disorders and were diagnosed with either PTSD [13], moderate to severe anxiety [14] or a moderate to severe depressive [15] disorder during the post-month from the day of the interview. None of the control participants showed any stress related affective disorders. In addition, the individuals with prescription opioid use disorder showed an increased perceived stress score during the last month indicating a higher perceived stress compared to the healthy controls ( $\mathrm{t}$-test, $\mathrm{p}<0.1$ ). PO addicted individuals' mean perceived stress score fell into the moderate stress category whereas the control participants' stress level was low and the mean score fell into the low stress category. Taken together, these results demonstrate that ACEs are strongly associated with abuse of prescription medications during the adulthood years. In addition, individuals with multiple adverse events during childhood may be more prone to develop comorbid opioid addiction and psychiatric 
disorders such as depressive or anxiety disorder in their adult years.

They furthermore experienced a heightened perceived stress during the past month. These findings emphasize an enhanced need to comprehensively address untreated and undiagnosed mental health issues among those with a history of trauma as a public health priority. This is particularly important as stress related disorders or an enhanced perceived stress in PO addicted individuals may contribute to an increased use of opioids or a greater risk of relapse to opioids after addiction treatment. Thus, it is crucial to utilize interventions that can target the stress symptoms in the opioid patients. One of these interventions may include heart rate variability biofeedback $[16,17]$, a bio-behavioral intervention that targets the neurophysiological component of affect regulation and craving. If proven efficacious, it can be provided as part of the current first-line treatments for opioid users suffering from stress related affective disorders or chronic perceived stress.

In addition, since the ACEs are a serious public health concern, the communities should work together with the families in order to prevent child maltreatment and produce positive physical and mental health outcomes for the children and families. The public schools in the communities may promote a brief stress management intervention for students in the class specifically for kids dealing with adversity in order to ensure that they can better regulate stress and negative emotion. This proposal is consistent with a study conducted by Bothe and colleagues (2014) who demonstrated that teacher-led daily 10-minute stress management intervention improved elementary students' anxiety and they were better able to handle their stress at school and at home.

\section{Acknowledgements}

This research was supported by a National Institute on Drug Abuse grant (R03DA044496) to Dr. Suchismita Ray. The author is thankful to Mr. Robert Budsock, the CEO of Integrity House and Mr. Thomas Lusch, the Senior Director of Integrity House for allowing her to recruit individuals with prescription opioid use disorder from their facility for this research. Finally, the author is grateful to the patients whose data were used in this report.

\section{Conflict of Interest}

The author has no conflict of interest to declare.

\section{References}

1. Back SE, Gros DF, Price M, La Rowe S, Flanagan J, et al. (2015) Laboratoryinduced stress and craving among individuals with prescription opioid dependence. Drug and alcohol depend 155: 60-67.
2. Florence C, Luo F, Xu L, Zhou C (2016) The economic burden of prescription opioid overdose, abuse and dependence in the United States, 2013. Med care 54(10): 901-906.

3. Bose J, Hedden SL, Lipari RN, Park-Lee E, Porter JD, et al. (2016) Key substance use and mental health indicators in the United States: results from the 2015 National Survey on Drug Use and Health. Department of Health and Human Services.

4. Feingold D, Brill S, Goor-Aryeh I, Delayahu Y, Lev-Ran S (2018) The association between severity of depression and prescription opioid misuse among chronic pain patients with and without anxiety: A crosssectional study. J Affect Disord 235: 293-302.

5. Khan S, Michmizos K, Tommerdahl M, Ganesan S, Kitzbichler MG, et al. (2015) Somatosensory cortex functional connectivity abnormalities in autism show opposite trends, depending on direction and spatial scale. Brain 138(5): 1394-1409.

6. Quinn K, Boone L, Scheidell JD, Mateu-Gelabert P, McGorray SP, et al. (2016) The relationships of childhood trauma and adulthood prescription pain reliever misuse and injection drug use. Drug Alcohol Depend 169: 190-198.

7. Perry BD, Pollard R (1998) Homeostasis, stress, trauma, and adaptation: A neurodevelopmental view of childhood trauma. Child Adolesc Psychiatr Clin N Am 7(1): 33-51.

8. Dunn EC, Nishimi K, Powers A, Bradley B (2017) Is developmental timing of trauma exposure associated with depressive and post-traumatic stress disorder symptoms in adulthood? J Psychiatr Res 84: 119-127.

9. Dworkin ER, Ojalehto H, Bedard-Gilligan MA, Cadigan JM, Kaysen D (2018) Social support predicts reductions in PTSD symptoms when substances are not used to cope: A longitudinal study of sexual assault survivors. J Affect Disord 229: 135-140.

10. McHugh RK, Gratz KL, Tull MT (2017) The role of anxiety sensitivity in reactivity to trauma cues in treatment-seeking adults with substance use disorders. Compr Psychiatry 78: 107-114.

11. Felitti IVJ, Anda RF (2007) Adverse childhood experiences and stress: paying the piper. ACE reporter.

12. Cohen S, Kamarck T, Mermelstein R (1983) A global measure of perceived stress. J Health Soc Behav 24(4): 385-396.

13. Weathers FW, Litz BT, Keane TM, Palmieri PA, Marx BP, et al. (2013) The ptsd checklist for dsm-5 (pcl-5). Scale available from the National Center for PTSD.

14. Hamilton MAX (1959) The assessment of anxiety states by rating. Br J Med Psychol 32(1): 50-55.

15. Beck AT, Steer RA, Brown GK (1996) Beck depression inventory-II. San Antonio 78(2): 490-498.

16. Karavidas MK, Lehrer PM, Vaschillo E, Vaschillo B, Marin H, et al. (2007) Preliminary results of an open label study of heart rate variability biofeedback for the treatment of major depression. Appl Psychophysiol Biofeedback 32(1): 19-30.

17. Lehrer PM, Gevirtz R (2014) Heart rate variability biofeedback: how and why does it work? Frontiers in psychol 5: 756. 